\title{
Special feature: spatial statistics
}

\section{Yasumasa Matsuda ${ }^{1}$}

Published online: 25 May 2020

(C) Japanese Federation of Statistical Science Associations 2020

It is possible to regard statistics as a trial to analyze dependency in data. Starting from analysis under independency, statistics has been developing for dependent observations, and dependency can be seen in diverse fields of data. Among them, time series dependency has attracted great interest for many years, where stochastic process theories helped lead to development in a rigorous way. On the other hand, spatial dependency has received less attention than time series, and spatial data analysis has been developed independently from the mainstream of statistics in an ad hoc way for practical issues in several areas such as mining and forestry. One reason for the difference in time series and spatial data is in the easier recognition of time than of space. The global flow of time from past to future is easily recognized by humankind, and events have been recorded in time as history in many traditional societies, while recognition of space is less concrete than that of time. The axis of the prime meridian, or longitude $0^{\circ}$, to express global location was established in 1871, much later in comparison with the Gregorian calendar, introduced in 1582.

Spatial and/or space-time problems in a wide range of areas have attracted more and more attention especially after 2000, with a proliferation of books, conferences, and papers. The explosion of interest in spatial statistics has been largely fueled by the increased availability of large spatial and patios-temporal datasets across many fields, as from the recent progress in geographic information systems (GIS). Spatial statistics can be regarded as one of the most critical areas in statistics to work for many modern issues in the age of big data.

Spatial statistics are recognized as having three major branches: (1) continuous spatial variation, (2) discrete spatial variation, and (3) spatial point pattern. This special issue of the journal includes 14 significant contributions in several areas that are categorized in those three major branches. In particular, let us introduce Noel Cressie, Peter Robinson, and Yoshihiko Ogata as the world-famous researchers leading the three branches. We do hope readers enjoy the recent progress of spatial statistics seen in their papers.

Yasumasa Matsuda

yasumasa.matsuda.a4@tohoku.ac.jp

1 Graduate School of Economics and Management, Tohoku University, Sendai, Japan 
Finally, we close this preface by introducing two major discoveries that have accelerated modern progress in spatial statistics. We believe this historical introduction helps provide an overview of how it began.

The first one is the Matérn class proposed in a doctoral dissertation (1960) by Bertil Matérn. Matérn used the class of models for the correlation structure of spatially continuous stationary processes that is now named the Matern class, which takes the form

$$
\rho(u)=\left\{2^{\kappa-1} \Gamma(\kappa)\right\}^{-1}(u / \phi)^{\kappa} K_{\kappa}(u / \phi),
$$

where $\phi$ is a scale parameter and $K_{K}$ denotes a modified Bessel function of order $\kappa$. The parameter $\kappa$, which is poorly identified empirically by a spatial dataset, describes the smoothness of the process that can provide useful information in forecasting performances. The Matérn class is a remarkable model in geostatistics that remains very much employed for kriging. Diggle (2010) introduced the interesting story of how the doctoral thesis came to be so widely cited. He learned from Sir David Cox that Matérn had visited London in the late 1960s to give what turned out to be a very influential series of lectures, after which copies of the dissertation began to circulate within the fledgling community of spatial statistics researchers in the United Kingdom.

The other major discovery is the conditional autoregressive (AR) models by Julian Besag, who initiated (1974) a systematic account for lattice data, spatial data observed on grid points. His critical contributions are discriminations between conditional and simultaneous AR models on a lattice, which are identical in time series cases. Let us introduce a simple case of a Gaussian AR time series model, which is defined by, for iid Gaussian $\varepsilon_{t} \sim \operatorname{iidN}\left(0, \sigma^{2}\right)$,

$$
X_{t}=\phi X_{t-1}+\varepsilon_{t}, \quad t=1, \ldots, T,
$$

which satisfies

$$
X_{t} \mid\left\{X_{s}, s \leq t-1\right\} \sim N\left(\phi X_{t-1}, \sigma^{2}\right) .
$$

Similarly let us introduce a spatial model on a one-dimensional lattice, which is defined by, for iid Gaussian $\varepsilon_{i} \sim \operatorname{iidN}\left(0, \sigma^{2}\right)$,

$$
X_{i}=\phi\left(X_{i-1}+X_{i+1}\right)+\varepsilon_{i}
$$

which does not satisfy

$$
X_{i} \mid\left\{X_{j}, j \neq i\right\} \sim N\left(\phi\left(X_{i-1}+X_{i+1}\right), \sigma^{2}\right) .
$$

The discrimination of the conditional and simultaneous AR models in spatial statistics promoted two ways of development. One is directed to Bayesian hierarchical models, such as in epidemiology and ecology, while the other has led to spatial econometrics, a new area in that field.

We do hope readers will enjoy learning of the recent progress in spatial statistics presented in this special issue, originating in the two key papers introduced here. 


\section{References}

Besag, J. E. (1974). Spatial interaction and the statistical analysis of lattice systems (with discussion). Journal of the Royal Statistical Society B, 36, 192-225.

Diggle, P. J. (2010). Historical introduction. In A. E. Gelfand, P. J. Diggle, M. Fuentes, \& P. Guttorp (Eds.), Handbook of spatial statistics (pp. 3-14). Boca Raton: CRC Press.

Matérn, B. (1960). Spatial variation. Meddelandenfran Statens Skogsforsknings Institute, Stockholm, Band 49, No. 5.

Publisher's Note Springer Nature remains neutral with regard to jurisdictional claims in published maps and institutional affiliations. 